\title{
Protée
}

\section{De la peinture de ruines à la ruine de la peinture. Hubert Robert et le Louvre}

\section{Johanne Lamoureux}

Volume 27, numéro 3, 1999

L’imaginaire de la fin

URI : https://id.erudit.org/iderudit/030571ar

DOI : https://doi.org/10.7202/030571ar

Aller au sommaire du numéro

\section{Éditeur(s)}

Département des arts et lettres - Université du Québec à Chicoutimi

ISSN

0300-3523 (imprimé)

1708-2307 (numérique)

Découvrir la revue

Citer cet article

Lamoureux, J. (1999). De la peinture de ruines à la ruine de la peinture. Hubert Robert et le Louvre. Protée, 27(3), 56-69. https://doi.org/10.7202/030571ar

\section{Résumé de l'article}

Il semblerait que le discours historique et critique sur l'art en Occident, avan même l'iconoclasme et l'aniconisme de tableaux modernes, ait d'entrée de jeu été travaillé par le ressassement de la mort imminente de la peinture. Cet article veut montrer comment cette problématique - l'impossible survivance de la peinture - a pu être aussi formulée par la peinture même. Il s'y emploie en s'attardant à deux oeuvres d'Hubert Robert (1733-1808) où des conceptions opposées de l'histoire, progressiste ou cyclique, sont traduites spatialement : Le Projet d'aménagement de la Grande Galerie du Louvre et son pendant La Vue imaginaire de la Grande Galerie du Louvre en ruines (1796). Ce dernier tableau relève l'échec de la fonction de conservation du musée puisque la peinture en a disparu sans laisser d'autres traces que sculpturales, pointant peut-être ainsi l'incurable destin moderne de ce medium. Mais déjà le premier tableau de la paire, par le pivotement de l'axe latéral du récit sur la profondeur de l'axe discursif et énonciatif des tableaux, substituait à la peinture d'histoire une histoire de la peinture dont cette nouvelle invisibilité des oeuvres semble avoir été le prix à payer. 


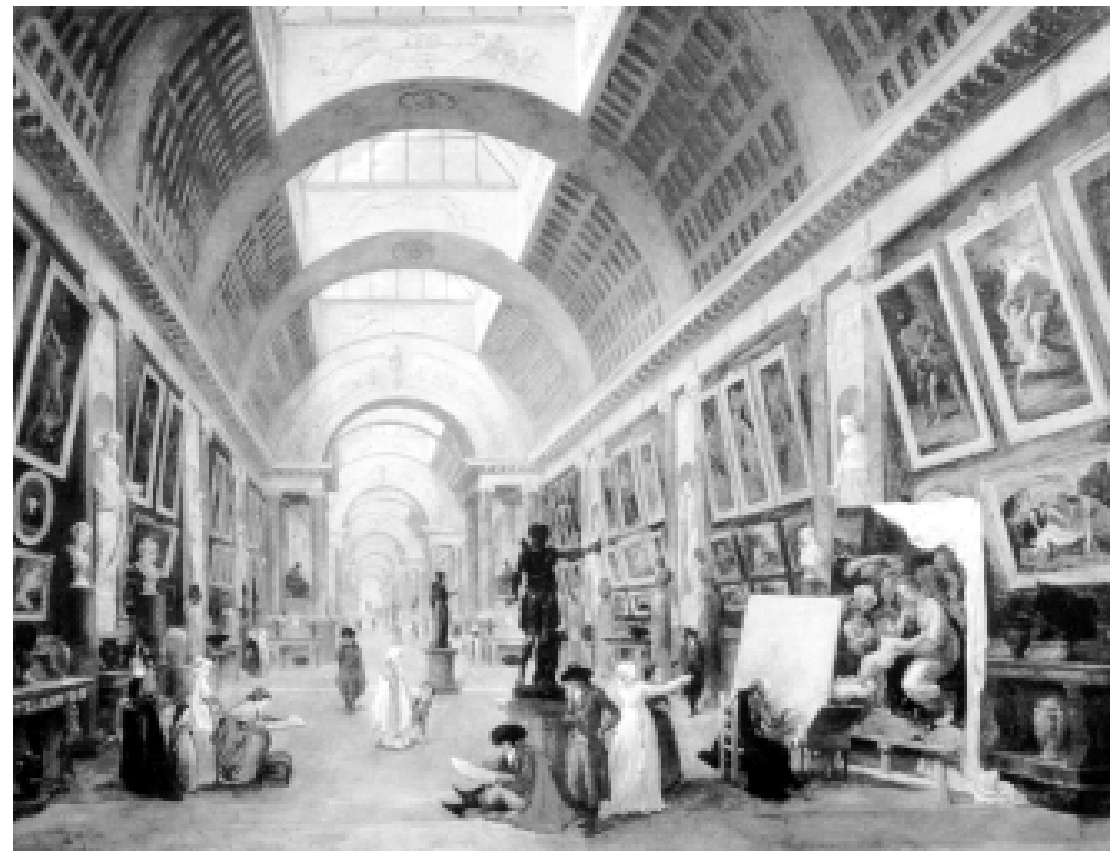

Robert Hubert, Projet d'aménagement de la Grande Galerie du Louvre, 1796, Paris, Louvre.

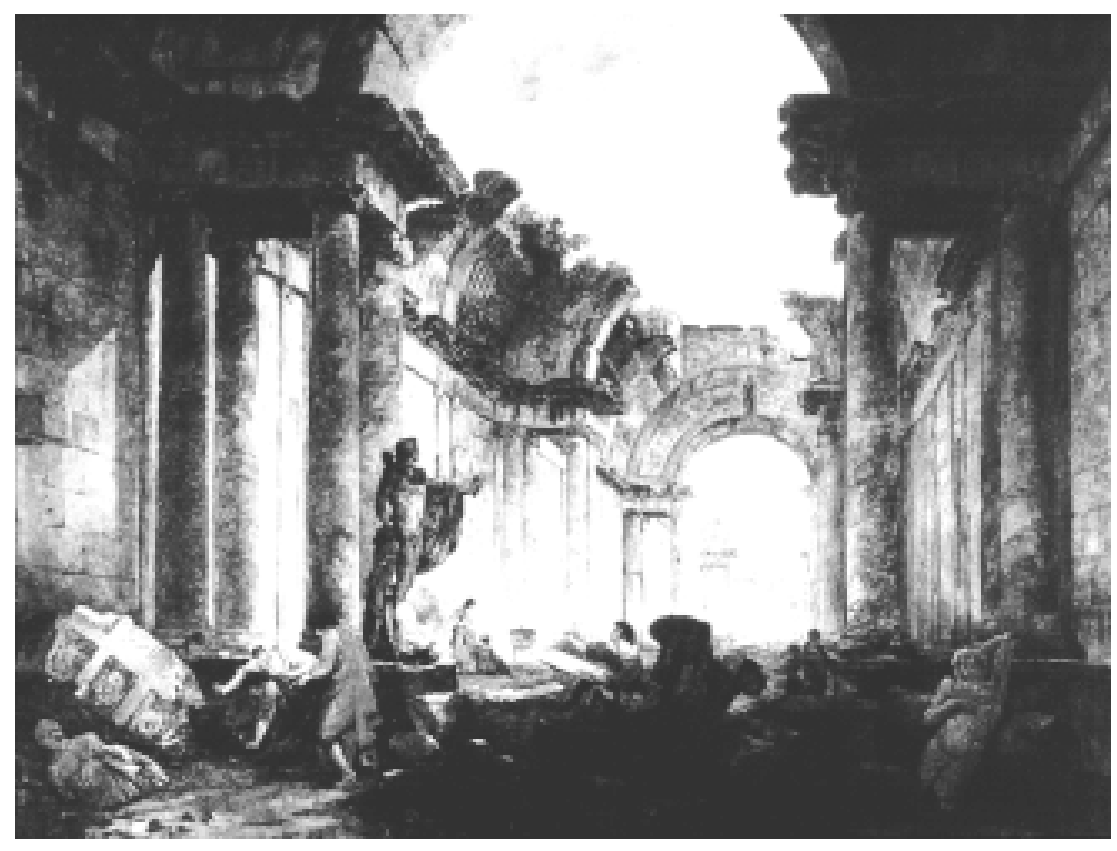

Robert Hubert, Vue imaginaire de la Grande Galerie dans les ruines du Louvre, 1796, 114,5 x 146cm, Paris, Louvre. 


\section{DE LA PEINTU RE DE RU INES À LA RUINEDE LA PEINTURE HUBERT ROBERT ET LE LOUVRE}

JOHANNE LAMOUREUX

L'imaginaire de la fin, tout historien de l'art devrait s'en croire expert, si tant est que le pictocentrisme de l'histoire de l'art lui aura ouvert les yeux sur le fantasme du dernier tableau et de la peinture morte qui ressurgissent, travestis et métamorphosés, de Giorgio Vasari à Daniel Buren. Pourtant l'idée que l'imminence de la fin est un trait - un symptôme - récent de l'histoire de la peinture est assez bien ancrée. Ainsi, on peut lire dans un texte d'Yve-Alain Bois intitulé «Painting: The Task of Mourning»:

Nothing seems more common in our present situation than a millenarianist feeling of closure. Whether celebratory (what I will call manic) or melancholic one hears endless diagnoses of death: death of ideologies (Lyotard), of industrial society (Bell), of the real (Baudrillard); of authorship (Barthes); of man (Foucault); of history (Kojeve) and, of course, of modernism (all of us when we use the word postmodern). Yet what does all of this mean? From what point of view are these affirmations of death being proclaimed? ${ }^{1}$

Évoquant Derrida, Bois précise aussitôt qu'il ne saurait y avoir de réponse générique à cette question.

There is no single paradigm of the apocalyptic, and no ontological inquiry about its tone. Because the tone of their writings is so different, it would be particularly misguided and perverse, to connect Barthes to Baudrillard; Foucault to Bell, Lyotard to Kojeve - but it is done in the theoritical potpourri one reads month after month in the flashy magazines of the art world. [...] in each instance, one must examine the tone of the apocalyptic discourse; its claim to be the pure revelation of truth, and the last word about the end. I will focus here on a specific claim; that of the death of painting, and more specifically the death of abstract painting.

Le texte de Bois est une riposte à "The End of Painting "², un article paru une décennie plus tôt sous la plume de Douglas Crimp. Ce dernier y défendait une formulation du postmodernisme conçu comme l'abolition de l'autonomie de la sphère esthétique et rappelait les coups marqués envers celle-ci par le travail iconoclaste de Daniel Buren, qui, il y a trente ans, répudiait le code désuet de la peinture et décidait de limiter ses interventions à une indexation des conditions de possibilités matérielles et institutionnelles de la production artistique. Face à de 
telles positions, Bois rappelle que la mort de la peinture ne saurait néanmoins caractériser la fin du modernisme dans la mesure où cette obsession se trouve déjà inscrite dans les premiers balbutiements de la peinture abstraite. Le modernisme pictural, insiste Bois, n'aura pas été autre chose que ce commencement de la fin ${ }^{3}$, «être moderne» se résumant après tout, selon Barthes, «à savoir ce qui n'est désormais plus possible ${ }^{4}$. À le savoir ou à l'imaginer?

Plusieurs faits donnent raison à Bois et nous invitent à hausser les épaules devant la dernière mort en date de la peinture. Malevich écrivait en 1920: « La peinture a depuis longtemps fait son temps et le peintre lui-même est un préjugé du passé» ${ }^{5}$. On peut aussi songer à la proclamation de Nicolas Taraboukine autour des trois monochromes de Rodchenko peints en 1921:

Chaque fois qu'un peintre a voulu se débarrasser réellement de

la représentativité, il ne l'a pu qu'au prix de la destruction de la peinture et de son propre suicide en tant que peintre. Je pense à une toile récemment proposée par Rodchenko à l'attention des spectateurs. C'était une petite toile presque carrée entièrement couverte d'une unique couleur rouge. Cette ouvre est extrêmement significative de l'évolution subie par les formes artistiques au cours des dix dernières années. Ce n'est plus une étape qui pourrait être suivie de nouvelles autres, mais le dernier pas, le pas final effectué au terme d'un long chemin, le dernier mot après lequel la peinture devra se taire, le dernier "tableau» exécuté par un peintre. ${ }^{6}$

Si on ajoute à cela la belle démonstration menée par Thierry de Duve à propos de la généalogie picturale du ready-made inventé par Duchamp à peu près à la même époque ${ }^{7}$, (le ready-made serait une négation de la peinture-métier) ou les prophéties de Malevich et celles de Mondrian: «la peinture deviendra absorbée par la vie» ${ }^{8}$, il semble que la position de Bois soit juste et qu'un certain postmodernisme ait vite condamné la peinture, sous prétexte que les exigences essentialistes et l'opération historiciste du modernisme de Clement Greenberg 9 en avaient précipité l'épuisement comme forme exemplaire de la haute culture. Mais voilà, si elle ne relève pas d'une situation causée par le postmodernisme, la mort de la peinture ne saurait non plus, comme le veut Bois, être envisagée comme un leitmotiv de la seule modernité, à moins d'élargir cette modernité fort au-delà des confins de l'histoire de l'abstraction picturale. C'est un projet auquel la colossale étude sur la peinture monochrome de Denys Riout s'est admirablement mesurée, enfonçant les racines de la monochromie dans le XIX'e siècle ${ }^{10}$. Pour Riout, la mort de la peinture semble indissociable de pratiques iconoclastes ou aniconiques; chez Bois, la question paraît davantage liée à l'émergence de la reproductibilité technique, alors qu'il me paraît indéniable que les textes sur la peinture et les œuvres figuratives entretiennent, très tôt dans la tradition du commentaire pictural en Occident, la peinture dans une situation d'agonie, dans une situation extrême où pointe toujours l'horizon de la fin. De Pline à Delaroche devant son premier daguerréotype ("la peinture est morte à dater de ce jour»), la mort imminente de la peinture a en effet constitué un des leitmotive les plus sous-estimés de l'histoire de l'art occidentale ${ }^{11}$.

Il se pourrait bien que le dénominateur commun de toutes ces morts déplorées, anticipées, exaltées de la peinture se trouve dans le traumatisme d'une peinture antique rêvée et jamais retrouvée, du moins pas telle qu'on l'avait fantasmée. C'est le sentiment qui se dégage d'une lecture serrée des textes et des images de la seconde moitié du XVIIIe siècle. Déjà, au début du XVIIe siècle, Rubens, prévenu par le sort de la peinture antique, proposait:

Nous n'avons pas d'autre moyen pour nous représenter les ceuvres des peintres de l'antiquité, que notre imagination... Je voudrais donc [...] qu'on imprimât un traité où seraient reproduits des tableaux de maîtres italiens, puisque des exemples de leur art sont encore visibles aujourd'hui, et qu'on peut les montrer du doigt en disant: les voilà. 12

Au cours du siècle suivant, devant la relative déception alors causée par les fragments picturaux retrouvés dans les sites archéologiques de Pompéi et 
d'Herculanuum, de même que devant l'impossibilité de dresser une taxinomie de la peinture antique équivalente à celles qui, au même moment, étaient établies pour la sculpture et l'architecture, le thème de la mort de la peinture refait surface. Mais il n'est plus associé cette fois à des visées rhétoriques, comme chez Vasari évoquant la peinture mourant avec Raphaël, ou à des considérations stylistiques, comme chez Poussin («la pauvre peinture réduite à l'estampe [et] à la sépulture»13). Cette fois, le thème sera décidément alimenté par une inquiétude plus strictement matérielle. La peinture est un medium auquel la précarité des matériaux semble interdire la survie dans le temps, autrement que sous forme d'anecdotes ou sous la forme légendaire du nom propre. Elle paraît en quelque sorte condamnée à la modernité.

Les textes de la fin du XVIIIe siècle témoignent souvent de cette douloureuse précarité de la peinture. Mais il se trouve que des tableaux de l'époque ont aussi manifesté des préoccupations du même ordre, sans qu'on l'ait jusqu'ici souligné. Le présent texte voudrait s'intéresser à deux d'entre eux: Projet d'aménagement de la Grande Galerie du Louvre et son pendant Vue imaginaire de la Grande Galerie du Louvre en ruine ${ }^{14}$. Ces deux ouvres d'Hubert Robert, dit «Robert des ruines», furent exposées au Salon de 1796 alors que le peintre siégeait au Conservatoire assurant la gestion du nouveau Muséum, entrevu par la monarchie et inauguré par la Révolution. Comme chez Gian Paolo Panini, un peintre ruiniste dont s'inspira Robert pour cette paire de tableaux, le genre, alors très populaire de la peinture de ruines, semble avoir servi à élaborer une singulière mise en signes de la ruine de la peinture et une réflexion picturale sur la possibilité que la peinture ne puisse, tout compte fait et malgré la promesse de l'institution naissante qu'est alors le musée, survivre au temps.

C'est ce chiasme entre la peinture de ruines et la ruine de la peinture que je voudrais étudier autour de ces deux tableaux. La nature de mon hypothèse sur les morts de la peinture occidentale ne se prête pas facilement à un développement en survol qui tendrait à confondre des motivations souvent fort différentes derrière les arrêts de mort ou les agonies proclamées du medium: il me semble donc plus prudent de considérer en premier lieu une série de cas qui devraient d'abord me permettre de singulariser les diverses occurrences de ce phénomène. Le présent article se veut une telle étude, et encore est-il présenté ici dans une version abrégée qui ignore délibérément des textes contemporains, notamment ceux de l'Encyclopédie (Diderot et d'Alembert, 1751-1780) et les travaux autour des découvertes d'Herculanuum et de Pompéi, au profit d'une analyse des œuvres. Il s'agira de faire apparaître comment la scénographie de la paire et le dispositif de chacun de ces tableaux annoncent le sort fatal du medium qui les a rendus possibles.

Dans un premier temps, on pourrait croire que les deux œuvres d'Hubert Robert articulent davantage leur problématique ruiniste à celle du musée, ou de la collection. Au moment où Hubert Robert les peint, il pratique déjà le genre ruiniste depuis une quarantaine d'années et sa peinture a suscité, en 1767 , les très belles pages de Diderot sur la poétique des ruines. Agréé à l'Académie, en 1766, comme peintre d'architecture, il connaîtra une belle carrière de décorateur, de jardinier, et bientôt de conservateur. Faut-il s'étonner que ce soit en effet à un tel artiste, qui n'était point un de ces peintres d'histoire auxquels incombaient habituellement de semblables honneurs, que l'intendance culturelle de Louis XVI ait confié la charge de gardien des collections royales, dès 1777 , puis à partir de 1784, de responsable du Muséum à venir. (On lui adjoindra néanmoins Jollain, un peintre d'histoire, pour faire taire les gazettes.)

Il faut bien comprendre aussi que selon la hiérarchie des genres qui règne encore à l'Académie, un peintre de ruines a un statut quand même supérieur à un peintre d'architecture, et ce pour des raisons esthétiques et éthiques. La ruine est perçue comme un objet plus propice à la peinture, un objet plus "pittoresque», comme on le dit alors, car les ravages du temps se traduisent le plus souvent par une asymétrie des formes et une irrégularité des contours, alors que les édifices récents constituent des référents 
dont la symétrie, redoublant celle des tableaux, risque de produire des œuvres ennuyeuses. «La symétrie, essentielle dans l'architecture, est bannie de tout genre de peinture", avance Diderot dans ses Pensées détachées ${ }^{15}$, précisant dans le Salon de 1767 qu' «il faut ruiner un palais pour en faire un objet d'intérêt». Les ruines sont nécessairement des édifices monumentaux, mais elles sont plus intéressantes que les monuments intacts car elles sont susceptibles d'inspirer des considérations philosophiques sur les leçons de l'histoire. D'un côté, elles font office d'opérateur interdisciplinaire; elles rendent compatibles l'architecture et la peinture; de l'autre, elles mettent l'architecture en histoire, elles en présentent une version condensée, elliptique, indicielle (Peirce les incluait d'ailleurs dans sa nomenclature d'index ${ }^{16}$ ).

Après la Révolution, Robert réussit à maintenir cette charge institutionnelle et fera partie du Conservatoire qui, sous le Directoire, assure la gestion du Muséum nouvellement ouvert. Durant ces années, l'enchevêtrement de la ruine et du musée apparaît relativement banal, malgré la nouveauté de l'institution, et ce phénomène peut s'expliquer de deux manières. En premier lieu, une certaine configuration épistémologique entre archéologie, histoire de l'art et muséologie favorise ce rapprochement. L'engouement archéologique qui traverse le siècle, après les découvertes de Pompéi et d'Herculanuum, tend vers le musée. Notamment en ce qui a trait aux fresques, souvent décevantes, qu'on y a mises au jour, la précipitation et le manque d'expertise avec lesquels on les expose à l'air et on les déplace, sont vus comme ruinant d'un coup ce qui avait survécu dix-sept siècles:

[...] les peintures antiques [ayant] le malheur que, du moment qu'elles voient le jour, l'air dont elles ont été privées pendant une longue suite de siècles les frappe et les dévore; la couleur s'éteint; et sans qu'on ait pu y trouver encore de remède, la peinture disparaît entièrement. 17

En second lieu, le contexte politique des premières campagnes napoléoniennes génère un butin considérable qui se trouve alors transporté de Rome à Paris: des protestations s'élèvent sur ce pillage qui a pour but d'alimenter le Louvre, Muséum national, et d'assurer la grandeur de la France républicaine. Les Lettres au général Miranda de Quatremère de Quincy sont le plus éloquent exemple d'une dénonciation de ce déplacement des monuments ${ }^{18}$. Vingt ans plus tard, Quatremère allait une fois encore déplorer le démembrement des collections romaines en étendant sa condamnation jusqu'aux musées qui en sont l'horizon ${ }^{19}$ :

C'est donc détruire ce genre d'instruction [...] que d'en décomposer les parties comme on n'a cessé de le faire depuis vingt-cinq ans, que d'en recueillir les débris dans ces dépôts appelés Conservatoires. Par quel étrange contresens appelleraiton de ce nom ces réceptables de ruines factices qu'on ne semble vouloir dérober à l'action du temps que pour les livrer à l'oubli? Cessez copistes ignorants de trouver du plaisir dans ces ruines; oui celles du temps sont respectables, celles de la barbarie font horreur. Les ruines du temps, ces monuments de la fragilité sont la leçon de l'homme, les autres en sont la honte. Cessez surtout de nous vanter l'ordre et l'arrangement qui règnent dans ces ateliers de démolition.

On devine que, dans la perspective de Quatremère, le Projet d'aménagement de la Grande Galerie $d u$ Louvre réitère une apologie de ce que, lui, ne perçoit que comme un "atelier de démolition", rempli de «ruines factices». Le Projet serait l'incarnation diabolique de la ruine de l'art telle que l'orchestre l'institution muséologique naissante. (On notera qu'Hubert Robert, sensible à cette chaude actualité du déplacement contesté, détache de l'enfilade de la Galerie un tableau de Raphaël acquis par François 1er du vivant de l'artiste et non un tableau saisi à Rome.) La Vue imaginaire, elle, ruinerait le musée, malgré le titre ambigu sous lequel elle figure au livret du Salon de 1796 - Ruines, d'après le tableau précédent-, titre qui use de la synecdoque autorisée par l'article de l'Encyclopédie cité plus haut, («ruine se dit du tableau qui les représente») et qui énonce déjà le principe d'une possible contamination entre la ruine et le tableau. 
Or je voudrais soutenir ici que les deux tableaux ont un même propos, à savoir l'effacement, selon des modalités différentes, de la peinture par l'histoire. Cette similitude se trouve déguisée sous les conventions régissant la production de tableaux par paire. On s'entend généralement pour croire qu'Hubert Robert a réalisé le Projet dans le but de convaincre les instances concernées et certains de ses collègues conservateurs des vertus de l'éclairage zénithal pour l'exposition des collections. Par la suite, il aurait, nous dit-on, entrepris, un peu par habitude, de transposer son sujet en ruine. On pourrait aussi arguer que, automatisme pour automatisme, c'est moins celui de la pratique ruiniste qui lui suggère cette idée, que son expertise de peintre décorateur pour qui il est coutumier de concevoir des tableaux en pendants. Cette explication un peu condescendante du pendant ruiniste est typique du discours critique entourant la production de Robert, discours enserré entre deux équivoques interprétatives. L'équivoque archéologique veut qu'il ait représenté des ruines en fonction d'un référent romain précis identifiable (alors que comme l'a montré A. Corboz ${ }^{20}$, sa syntaxe et son vocabulaire architecturaux n'ont pas d'équivalent, à Rome ou ailleurs, et relèvent souvent de l'utopie). L'équivoque muséologique fait de ses tableaux une plate illustration de ses fonctions de conservateur et déclare que l'histoire du Louvre passe désormais par lui, ce qui constitue une forme de reconnaissance ambiguë pour un peintre.

En tant que paire de tableaux, les œuvres qui nous intéressent ici s'apparentent à une figure de la rhétorique littéraire que l'Encyclopédie commente sous l'appellation de "peinture double":

On appelle peinture double, celle qui consiste à présenter deux images opposées, qui jointes ensemble se relèvent mutuellement. [...] la double peinture reste d'un merveilleux effet pour le pathétique, mais cette adresse est une des plus grandes $d u$ Poète et de l'Orateur, il faut la savoir ménager, l'employer sobrement et à propos. 21

Les pendants picturaux, bien que non joints littéralement, favorisent souvent le contraste, et particulièrement ce qu'on pourrait appeler les "contrastes naturalisés": les saisons, les heures du jour, les variations météorologiques. Parfois cet appétit de contraste se transpose en termes biologiques par une sexualisation de la paire: les portraits des époux Montefeltre par Piero della Francesca ou ceux d'Angelo Doni et de sa femme par Raphaël attestent cette convention. Svetlana Alpers cite une paire de tableaux de Metsu représentant un homme écrivant une lettre et une femme recevant une missive ${ }^{22}$. Dans ce cas, la complémentarité sexuelle redouble celle des actions et le thème fait écho à la distance réelle, à l'entre-tableaux, qui sépare les deux œuvres. Dans le cas du Projet et de la Vue, il y a à la fois allusions cosmogoniques et complémentarité sexuelle doublée de gémellité. En effet, le Projet présente une statue de la déesse lunaire Diane et la Vue une représentation de l'Apollon du Belvédère à la fonction desquelles je reviendrai plus loin.

Les tableaux jumeaux paraîtront décliner tous deux le futur du musée, le futur éclairé, lumineux, immédiat du Projet et la face plus sombre d'un futur du futur dans la Vue. En réalité, cette double représentation du futur, bien qu'apparemment unifiante, cache deux conceptions absolument antagonistes de l'histoire, une version linéaire, progressiste à la Condorcet, une vision cyclique à la Gibbon ${ }^{23}$. C'est sur ce plan que se joue le pathétique de cette paire et que se mobilise sa pleine valeur de contraste, qui met littéralement en scène le jour et la nuit de l'histoire.

Il faut préciser encore avant de passer à l'analyse singulière des tableaux que leur composition et leur désignation dans le livret du Salon de 1796 donnent des indications assez claires sur l'effet recherché. Ainsi, des pendants peuvent s'exposer de deux manières qui ne sont point exclusives: soit, exposés en parangon, ils se répondent directement, liés par leur thème, leurs motifs ou le style, en rimant l'un avec l'autre; soit, exposés en parergon, ils pendent en marge d'un ou de plusieurs tableaux dont ils sont alors les faire-valoir, redoublant du même coup la clôture microcosmique de toute collection par leur mise en 
évidence d'une règle de symétrie. Ce principe réaffirme immanquablement l'autoréférentialité de l'accrochage: les tableaux qui y ont place paraissant davantage s'articuler autour d'une pliure spéculaire intrinsèque à leur propre espace de présentation que se poser en miroir du réel qu'ils sont censés représenter. Les pendants étudiés ici, bien qu'ils entretiennent le rapport antithétique de la peinture double, opèrent surtout en parangon. Le titre inscrit au livret du Salon indique en effet qu'ils durent être exposés côte à côte.

Cette proximité devait, lors de l'exposition de 1796, rendre plus frappant encore le contraste entre les deux œuvres. Aucun segment de tableaux, aussi court soit-il, ne venait tenir lieu du temps écoulé entre les deux futurs exposés. Tout se jouait dans l'espace qu'il faut imaginer bien étroit compte tenu des conventions d'accrochage au Salon - entre les deux tableaux. Or cet espace, cette faille sont encore soulignés (comme s'il s'agissait véritablement de l'endroit auquel la paire assigne son regardeur) du fait que s'y croisent, sans convergence résolue, les axes de fuite complémentaires de chaque tableau (légèrement vers notre gauche pour le Projet, radicalement vers notre droite dans la Vue). Cette élaboration de l'espace, de l'espace des tableaux comme de celui qui les sépare, met le spectateur en tension: elle contrarie la force aspirante de la perspective accélérée du Projet. L'intervalle entre les œuvres vient donc marquer une triple place: la place des œuvres que, selon la dimension syntaxique de l'accrochage, les pendants pourraient encadrer mais qui sont ici sacrifiées, la place où, selon la dimension pragmatique, la paire inscrit son regardeur, et la place par laquelle se figure, et combien en raccourci, l'articulation sémantique des deux tableaux. Il y a syncope dans le déroulement temporel suggéré, et par-delà cette ellipse, les pendants semblent renvoyer dos à dos, ou plutôt coude à coude, la continuité de l'histoire de la peinture (mise en scène par le Projet) et l'endurance de la peinture dans l'Histoire (réfutée par la Vue). Enfin, il faut dire aussi que la faille donne à voir le présent du lieu réel, les œuvres étant exposées in situ au Louvre, en 1796 comme aujourd'hui: elle présente et présentifie cette institution que les deux tableaux re-présentent au futur.

Il est difficile aujourd'hui de considérer ces deux œuvres comme une double déclinaison du futur, tant l'aménagement de la Grande Galerie a fini, au cours du XX ${ }^{\mathrm{e}}$ siècle, par rattraper le Projet et par le transformer en simple portrait. Ce détournement illustratif incite les spectateurs à s'émerveiller davantage devant l'audace sacrilège de la Vue imaginaire parce que celle-ci consacre en quelque sorte l'échec de la sauvegarde et de la conservation qui sont la raison d'être du musée. Pourtant on peut supposer qu'en 1796 , c'est le pendant ruiniste qui apparût plus banal qu'il ne le semble à nous, visiteurs du Louvre de Pei, avec son cœur greffé de verre pointu et ses façades revampées. Il y a au moins deux arguments pour soutenir une telle supposition.

1) Comme l'a montré Roland Mortier ${ }^{24}$, la poétique des ruines de la seconde moitié du XVIII siècle est anticipatrice plutôt que rétrospective; elle invite à faire, pour reprendre le titre d'un roman d'Antoine Compagnon, un «deuil antérieur». La dégradation et la réappropriation des monuments $\mathrm{du}$ passé par la force organique de la nature rappellent moins à ceux qui en contemplent les résultats le sort de ceux qui ont vécu au moment de cette gloire passée que la relative éphémérité des lieux qu'ils chérissent aujourd'hui et qu'ils savent néanmoins être voués à périr un jour ${ }^{25}$. En ce sens, le courant ruiniste de l'époque rejoint le genre utopique pratiqué au même moment, lequel, plutôt que d'imaginer les conditions idéales d'un monde du «nulle part», applique ces conditions à un lieu bien identifié mais précipité dans un futur plus ou moins défini ${ }^{26}$.

2) Le délabrement du Louvre s'apparente au lieu commun durant le XVIII e siècle (et peut-être même dès le XVII e siècle, si l'on en juge par les remarques de Poussin lors de son invitation à peindre le plafond de la Grande Galerie). Loin de surgir dans un ensemble architectural homogène, le palais est coincé dans un environnement urbain encombré de ruelles étroites et de constructions branlantes qui donnent aux abords 
de l'édifice un caractère labyrinthique au milieu duquel le monument paraît laissé à l'abandon. Les artistes qui se voient attribuer un logement au Louvre, comme le veut l'usage avant la conversion du palais en musée, se plaignent tous de l'état des lieux. L'article «Louvre» de l'Encyclopédie termine ainsi ses recommandations sur l'avenir du palais: "Voilà ce qu'il serait beau de faire de ce vaste édifice, qui peutêtre dans deux siècles n'offrira plus que des débris " 27 . Mercier, dans L'An 2440 ou rêve s'il en fut, inaugure son récit par une dénonciation de l'état du Louvre au moment où il rédige ces premières lignes (1770): «Soyez fiers de tous vos beaux monuments qui tombent en ruine: montrez avec admiration votre Louvre, dont l'aspect vous fait plus de honte que d'honneur " ${ }^{28}$. Enfin, la banalité du Louvre en ruine transparaît jusque dans ce commentaire d'un visiteur du Salon de 179629:

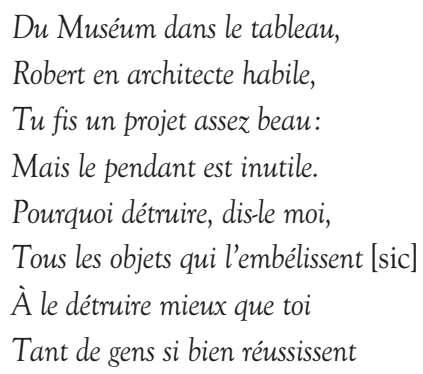

Or Robert aura jugé, lui, que le pendant avait son utilité, à cause peut-être de la fonction d'autorité par laquelle l'anticipation ruiniste sert à "vendre» la solution architecturale proposée dans le Projet en la voyant s'accomplir, par-delà sa réalisation, dans une noble décrépitude d'inspiration romaine, en promettant au Paris de demain la fortune archéologique de la Rome d'alors. La nouveauté un peu choquante (et techniquement encore utopique) de l'éclairage zénithal est assourdie en tant que s'y profile, morphologiquement, le contour de la ruine à venir. La ruine, dont la voûte effondrée constitue le motif quasi emblématique au XVIII siècle, est dès lors déjà inscrite dans le filigrane du Projet: elle s'y trouve programmée par la verrière. Robert ne projette pas des ruines: il ruine des projets.
Néanmoins, le recours à un tel argument d'autorité ouvre un paradoxe. La perspective en tunnel du Projet qui, comme l'a vu Corboz, incarnait de manière fulgurante la définition laconique $\mathrm{du}$ progrès selon l'Encyclopédie - «mouvement vers l'avant» -, semble se révulser, par-delà la faille entre les tableaux, en un futur passéiste, désormais étayé sur une vision cyclique de l'histoire. Condorcet, dans son Esquisse d'un tableau historique des progrès de l'esprit humain, affichait son mépris envers une telle conception de l'histoire par un parti pris délibérément anti-romain. Il y dénonçait le thème historique de la grandeur et de la décadence, n'y voyant qu' «un système pusillanime et corrompu qui condamne [le genre humain] à d'éternelles oscillations " 30 . Or le Projet, malgré son titre initial - Projet pour éclairer la galerie du Musée par la voûte, et la diviser sans ôter la vue de la prolongation du local -, n'est pas qu'une simple démonstration illustrative d'une solution architecturale un peu audacieuse: Robert y présente l'ordonnancement de la galerie en accord avec la conception de l'histoire prévalant dans l'administration postrévolutionnaire du Directoire, et celle-ci est proche des vues tabulaires de Condorcet, ainsi qu'en témoigne un rapport présenté au Conservatoire du Musée par Varon au printemps 1796. Ce rapport précise que les tableaux de la Grande Galerie devront être présentés par écoles et offrir «une suite ininterrompue des progrès de l'art et des degrés de perfection où les ont portés tous les peuples qui les ont successivement cultivés " ${ }^{31}$.

Dans cette perspective, la seule idée d'un pendant, indépendamment du désastre que la faille entre les tableaux condense elliptiquement, relève du sacrilège envers la conception dominante, progressiste, linéaire de cette histoire européocentrique. Il est vrai toutefois que, même pour Condorcet, si la continuité du progrès général de l'esprit humain ne fait pas de doute, la vision progressiste des arts s'avère une question épineuse; et cela, non point en vertu de ce qui faisait problème chez Vasari ou Perrault, c'est-àdire le sentiment d'une perfection atteinte qui invalide toute considération ultérieure de la 
perfectibilité humaine, mais à cause des décadences marquées qu'on y remarque, selon le canon encore absolu à l'époque du Beau antique, et à cause des limites que la contrainte d'imitation impose aux progrès de l'art. Bien que je me propose de démontrer ci-dessous à quel point, et à quel prix pour la peinture, le Projet épouse les recommandations du rapport Varon, la Vue imaginaire ne fait pas qu'opposer au premier tableau, sur le mode du contraste habituel à la rhétorique de la paire et à la double peinture, une conception de l'histoire antagoniste mais jugée également valable. Hubert Robert, il me semble, défend, dans le pendant, la thèse d'une véritable renaissance des arts à venir, par-delà la faille et la ruine du projet, et son propos s'appuie, paradoxalement étant donné que le site se veut une galerie de peinture, sur quatre sculptures «trouvées» au milieu des débris.

1) En bas, à gauche de l'œuvre, une Tête de Minerve en porphyre s'impose, selon Sahut et Garnier, comme «symbole, aux yeux des archéologues qui sont en train de la découvrir, de l'affectation primitive de la galerie au culte de la déesse des arts" 32 .

2) L'Apollon du Belvédère 33 figure à gauche de la Vue. S'il constitue une réponse antique à l'œuvre similairement disposée de Raphaël dans le Projet, du moins au point de vue de l'équilibre des masses de la composition, il demeure surtout le jumeau de la déesse Diane qu'on voit en tête du défilé de statues placées sur l'épine de la Galerie. Comme le dieu solaire est redécouvert ici, au milieu de la désolation du site assombri, alors que la déesse lunaire régnait sur une galerie irradiée de lumière, il faut comprendre que chaque statue jumelle insuffle dans l'œuvre où elle se trouve le principe du tableau opposé et que cette gémellité astrale, qui place chaque tableau sous une égide croisée, inscrit la paire du côté du cycle, plutôt que du côté de la série ou de la suite.

3) L'Esclave mourant de Michel-Ange permet ici à Robert de renforcer la notion de cycle, en évoquant un remake d'un des grands moments de l'histoire de la sculpture: la découverte du Laocoon, le 14 janvier 1506, laquelle sculpture fut identifiée par l'architecte
Sangallo (qu'on a rapporté s'étant fait accompagner par Michel-Ange pour l'occasion) comme le célèbre groupe antique décrit par Pline et allait bientôt supplanter la renommée de l'Apollon ${ }^{34}$. Les esclaves, commencés par Michel-Ange quelque temps après, dans le cadre du projet de tombeau jamais complété de Jules II, sont les premières statues de l'artiste à témoigner, par leur torsion, de l'influence de cette découverte que Robert avait déjà évoquée, dans un tableau de 1773, et située dans une vaste galerie ruinée. Si les connotations solaires de l'Apollon introduisaient dans la Vue en ruine les germes d'une renaissance, la statue de la Renaissance nous fournit le motif d'une répétition de l'histoire, répétition en boucle qui devait être d'autant plus frappante pour les contemporains de Robert que la statue se trouvait alors à l'entrée du Musée. L'Esclave mourant et qui paraît lutter pour s'extirper du sommeil de l'ensevelissement, c'est ici la figure du seuil au terme du musée. Il suggère une chaîne des arts (de l'antique à la Renaissance et de la Renaissance à l'avenir...) mais une chaîne qui a été à plusieurs reprises radicalement interrompue.

4) Un buste de Raphaël, exécuté par l'obscur sculpteur Rondoni, se trouve aux pieds de la statue d'Apollon. Il s'agit du seul objet répété dans les deux tableaux de la paire. Sa fonction est paradoxale. Elle indique Raphaël en le signifiant par son effigie bien connue, mais celle-ci sert surtout à montrer l'absence du grand chef-d'œuvre qui était à l'honneur dans le Projet. C'est Michel-Ange, le grand rival de Raphaël selon la prose vasarienne, qui désormais se mérite seul le privilège d'incarner l'art de la Renaissance.

On ne saurait être plus clair, les arts renaîtront des ruines de l'institution muséale, mais à la manière dont elles ont connu une première renaissance. De même que la grande peinture antique ne fut alors pas retrouvée, la grande peinture «moderne» (ainsi que Vasari désignait la peinture italienne de la Renaissance pour la distinguer de la manière grecque, byzantine) n'est pas vouée à devenir antique, malgré sa mise au musée. Ou plutôt si, elle deviendra antique en imitant le sort de cet idéal perdu: elle disparaîtra à 
son tour. Un autre petit tableau ruiniste de Robert, appartenant à la même série de travaux sur la Galerie du Louvre mais réalisé un peu après, nous montre des femmes squattant le musée désaffecté et alimentant un feu avec le bois des cadres de tableaux déjà disparus.

Que la peinture soit promise à un triste mais noble sort, on s'en convainc aisément dès lors qu'on remarque l'absence de tableaux dans la Grande Galerie en ruine (une évidence que les commentateurs ont souvent notée sans plus y réfléchir). Mais comment le Projet, aux allures plus optimistes et triomphantes, peut-il avoir incité un conservateur bien intentionné et un peintre astucieux à annoncer, sans en avoir l'air, un effacement des tableaux, qui ne sera pas cette fois leur dévoration par le temps mais bien plutôt leur disparition dans et par l'Histoire? C'est ce que je souhaiterais démontrer ici avant de conclure.

Les minutes des réunions du Conservatoire du Louvre permettent de mieux comprendre l'ampleur des tâches, à la fois grandioses mais souvent triviales à cause du manque de ressources, assignées aux premiers conservateurs: faciliter les travaux des copistes, préparer les événements spéciaux tels que le Salon et son livret, aménager les lieux à court terme et les imaginer à long terme, nettoyer la collection des faux et des œuvres médiocres qui s'y trouvent, améliorer la collection (par des achats, mais aussi par des saisies et par des échanges) et enfin disposer et présenter la collection pour la jouissance du plus grand nombre.

Selon le Conservatoire, la galerie devait s'ouvrir sur le hic et nunc de la suprématie contemporaine de l'École française, pour ensuite remonter vers le premier âge d'or de la peinture moderne, la Renaissance italienne, en passant par la tradition nordique. L'histoire de la peinture devait être donc racontée en flash-back, à partir de sa justification téléologique dans l'École française. C'est pour remettre cette histoire dans le bon sens, pour que le point de fuite inscrit là-bas, au fond du tunnel, réfère à un moment ultérieur à celui représenté de part et d'autre du point de vue du regardeur, que Robert situe ce dernier dans les travées italiennes, là où le parcours prévu devait se terminer: de cette façon, le point de vue (entendu ici dans le sens le plus général du terme, comme là d'où le regardeur est invité à regarder) et le point de fuite sont respectivement l'origine et le terme du cheminement historique des arts. Conceptuellement, il y a néanmoins un certain télescopage: le temps historique des premiers regardeurs des tableaux est contemporain de cette suprématie de l'École française suggérée autour du point de fuite. Robert privilégie ici le musée comme direction, comme accélération du sens de l'histoire et du destin de l'art, mais il le fait au détriment des tableaux représentés.

Au lieu de dresser l'inventaire de la collection en une façade d'architecture scénographique qui nous dévisagerait d'un coup et meublerait tout l'espace, Robert distribue les tableaux le long d'un axe architectural qui fuit à grande vitesse, en dérobant les tableaux à la vue et en menaçant de transformer les regardeurs en visiteurs: ces derniers sont invités à défiler devant le raccourci des œuvres alignées. Cette représentation de la pragmatique de l'exposition s'avère sans précédent à l'époque: il suffit pour s'en convaincre de rappeler brièvement, à travers quelques exemples célèbres, les formules antérieurement privilégiées dans la représentation picturale des collections de tableaux.

Dans La Visite aux Gobelins, Le Brun introduit un roi immobile, décentré sur la gauche: les plus beaux objets du dépôt lui sont présentés, comme si sa seule apparition venait de déclencher un réalignement orbital de ses collections et de son butin. Rien de comparable dans les tableaux, à peu près contemporains, où Teniers représente l'archiduc Léopold en promenade sur le terrain encore accidenté de sa collection, ostensiblement et méticuleusement figée sur les murs dans une véritable maçonnerie de cadres. Il arrive encore au siècle suivant que les tableaux et autres objets d'art soient déplacés, pour la montre, mais sur le mode d'une ponctuation insouciante du tableau. Qu'on pense par exemple à L'Enseigne de Gersaint de Watteau où des couples 
d'acheteurs potentiels distribués ici et là, c'est-à-dire sans constituer le centre solaire de la composition, se font présenter des tableaux d'une façon qui suggère que l'étalage commercial a déjà succédé à ce que Pierre Georgel appelle l'ostension sacrée ${ }^{35}$ du bel objet.

Par rapport à ces diverses propositions spatiales dont aucune ne compromet la visibilité des œuvres représentées et selon lesquelles les regardeurs du tableau sont admis à partager le privilège scopique du monarque ou du client, la solution retenue par Robert innove dans la mesure où l'artiste privilégie le dispositif en lieu et place de ce dont ce dispositif dispose, ou de ce qui trouve à s'y disposer. La Nation a remplacé les monarques et le discours historisant de l'institution naissante n'a que faire de l'étalage de collections somptueuses ou hétéroclites. Destinataire privilégiée de ce récit, elle impose ses conditions aux visiteurscitoyens et ne livre les précieux trésors de sa collection qu'à ceux qui acceptent d'y pénétrer, de faire corps avec la nouvelle institution, de participer à ce «mouvement vers l'avant» de la "République une et indivisible».

Cette organisation spatiale de la collection, et la circulation à laquelle elle nous engage formulent un désir nouveau de mise en perspective de la peinture qui est aussi un désir de coïncidence entre sa mise en perspective et sa mise en histoire: par-delà la tradition figurative des vues de collections où les tableaux, visibles, se trouvaient nivelés, littéralement mis sur le même plan, sans ordre repérable, la présentation impérieuse du Projet renoue, en les refaçonnant à cette fin, les liens, déjà bien établis par les galeries de portraits du XVIe siècle, entre le temps de l'histoire et la trajectoire d'un espace linéaire. Commentant les analyses de Philippe Ariès sur les galeries de portraits comme dispositif historique, Michel de Certeau écrivait de (et dans) L'Écriture de l'histoire ${ }^{36}$ :

L'écriture met en scène une population de morts - personnages, mentalités ou prix. Sur des modes et avec des contenus différents, elle reste liée à son archéologie $d u$ début $d u$ XVIIe siècle [...] à la galerie d'histoire telle qu'on la voit encore au chateau de

Beauregard: une suite de portraits, d'effigies ou d'emblèmes peints sur le mur avant d'être décrits par le texte organise le rapport entre un espace (le musée) et un parcours (la visite).
L'historiographie a la même structure de tableaux articulés par une trajectoire. Elle re-présente des morts le long d'un itinéraire narratif.

La galerie s'apparente aux mises en série classificatrices que l'épistémè du XVIIIe siècle finissant affectionne encore ${ }^{37}$, que ce soit sur le mode balbutiant des catalogues rédigés par les amateursmarchands, ou à travers les recueils archéologiques de monuments, d'ornements et d'ustensiles qui abondent en planches compilant les diverses variations d'un motif, les possibles déclinaisons d'un type. À l'instar de ces regroupements de nature plutôt tabulaire, la galerie retire l'œuvre du «réel», elle la «ruine» (dans le sens où Quatremère de Quincy conçoit tout déplacement muséologique), mais elle compense ce déracinement en lui garantissant, plutôt qu'un nouveau site, une nouvelle place au sein d'une série qui constituera désormais l'horizon combien «attractif» de ses significations. L'autonomisation de la sphère artistique se fonde précisément sur cette collusion entre l'histoire de l'art (et ses nouveaux modes de mise en série, ses nouvelles prétentions, après Winckelmann, à faire système) et le musée. La galerie, plus encore que la planche, la table ou toute autre forme de tabulation sédimentaire, est propice à ce dépaysement nécessaire au renforcement d'une histoire immanente des arts.

Les analyses de $\mathrm{K}$. Pomian ont montré avec force détails que toute collection, comme ensemble d'objets exposés à la vue, opère la transformation de l'invisible en visible ${ }^{38}$. Chaque objet exposé participe de ce processus de révélation; chacun témoigne du territoire exotique ou conquis dont il provient, de l'époque glorieuse révolue de laquelle il nous parvient ou du caractère miraculeux d'un épisode qui s'y trouve raconté. Mais qu'est-ce que la collection rend visible dès lors que les œuvres qui la constituent ne le sont pas? Peut-on dire alors que le tableau qui représente la collection la détourne de sa fonction du seul fait de cet effacement? Pomian nous fournit des éléments de réponse à cette question en rappelant que les objets, une fois au musée, tendent vers un invisible qui 
[...] n'est pas le même que celui dont ils sont originaires. Il est situé ailleurs dans le temps. Il s'oppose au passé, au caché et au lointain car il ne peut être représenté par quelque objet que ce soit. Cet invisible qui ne se laisse atteindre que dans et par le discours, c'est l'avenir. En mettant des objets dans les musées, on les expose au regard non seulement du présent mais aussi des générations futures, comme jadis on en exposait d'autres à celui des dieux. ${ }^{39}$

Cet invisible, nul autre tableau représentant une collection, avant ou après le Projet, ne réussit à le présenter aussi radicalement et avec tant de conviction. Au lieu que le tableau n'abrège la collection qu'il dépeint - et qui souvent se posait déjà elle-même comme monde abrégé -, le Projet la prolonge dans l'espace et dans le temps grâce à une enfilade d'œuvres en raccourci. À travers la durée imaginaire d'un parcours pourtant traversé d'un seul regard fulgurant, il rend visible, c'est-à-dire plus proche, là-bas dans le point de fuite et en toute instantanéité, l'avenir de l'art où, malgré l'illisibilité des œuvres, tout le dispositif spatio-temporel du Projet nous permet de «projeter» l'École française telle qu'en 1796 et au-delà.

Hubert Robert a fait en sorte que la profondeur de la perspective, dimension privilégiée de ces images, ne puisse être pensée comme une matrice, un réceptacle, de l'histoire mais opère comme son seul et unique vecteur de l'histoire et du tableau. Il rabat à cette fin l'axe traditionnellement latéral du récit classique (remis en vogue chez ses contemporains par les compositions en frise du néo-classicisme) et l'axe discursif (au sens de Benveniste) et énonciatif de la perspective ${ }^{40}$. Ainsi, l'espace représenté (le référent architectural de la galerie) devient, d'espace de présentation (la galerie comme musée) qu'il était, un ingénieux espace de re-présentation (la galerie comme dispositif historique), voire même un espace représentant (le tunnel comme traduction figurale d'une avancée progressiste de l'histoire dans laquelle le regardeur se trouve engagé par la perspective).

Devant ce dérobement des œuvres que ce dispositif entraîne, le vecteur de la galerie constitue leur seule liaison. Les tableaux se succèdent les uns aux autres, sans offrir, comme dans les formules antérieures, la possibilité d'un coup d'œil englobant sur les contenus de la collection. Seuls de petits personnages s'attardent ici et là en pauses admiratives et nous suggèrent la possibilité de dévisager les tableaux: ils nous tiennent lieu de seul regard autorisé. Au mieux, Robert favorise une rencontre plus ou moins oblique avec les œuvres (re)présentées. Il signe par conséquent le seul tableau vraiment visible de la collection (à l'exception de la Sainte Famille de Raphaël qu'il se représente en train de copier).

Avant même d'être, dans la Vue, réduite à un poudroiement de plus parmi les ruines, à un effet d'énonciation dans un tableau où elle ne figure plus comme énoncé, la peinture avait donc déjà été, dès le Projet, effacée par le dispositif de cette perspective muséographique qui s'empare de la peinture d'histoire pour en faire désormais l'histoire de la peinture. Dans l'ensemble du Projet, et particulièrement dans son lointain point de fuite, la peinture faisait doublement miroiter l'avenir, la gloire invisible de l'École française, et voilà que par-delà l'interstice qui sépare les pendants elle se trouve à détenir encore le privilège de pouvoir énoncer la scène apocalyptique où elle se déclare condamnée. Paradoxalement, la Vue... reste qui dit que la peinture n'a pas survécu, ne survivra pas, que l'art peut être antique mais que la peinture doit être moderne, ne trouvant à imiter son modèle antique que dans la fatalité qui la soustrait au jugement de la postérité et l'inscrit dans le mythe. En cela, ce tableau complexe venge le sort que l'histoire, au musée comme ailleurs, a réservé à la peinture, en produisant à son tour le futur ultime du musée sous la forme précaire d'un tableau, d'un tableau de ruines bien sûr, ou plutôt d'une ruine même, puisque « ruine se dit du tableau qui les représente».

Enfin, le tunnel de Robert, en machinant littéralement une substitution du référent de l'histoire, ouvre la voie à l'historicisme de la modernité. Remplaçant insensiblement la peinture d'histoire, si représentative de l'École italienne, par l'histoire de la peinture, il charge cette dernière de 
raconter comment la peinture française en viendra làbas, au terme de la galerie, à supplanter son modèle. Peu importe donc que la peinture française ne se laisse que deviner au loin dans la seule profondeur du profil de ses cadres, elle est de toute façon inutile dans son détail, dans la mesure où l'opération fondamentale du dispositif de la galerie est accomplie dès que l'on comprend que cette peinture d'histoire italienne, entrevue dans les premières travées sur les murs de droite, a désormais été relevée par l'histoire de la peinture. La hiérarchie des genres peut alors s'effondrer, comme le recommandait le rapport Varon ${ }^{41}$.

Clement Greenberg allait voir dans la peinture de Manet, ce peintre que Baudelaire proclama «le premier dans la décrépitude de [son] art», le moment fondateur du modernisme parce que l'histoire et l'anecdote y sont abolies au profit d'une affirmation de la planéité du support et de l'autoréférentialité du medium. Devant la traduction robertienne de l'historicisme muséologique naissant, on peut difficilement s'empêcher d'évoquer le commentaire de Rosalind Krauss sur la critique et l'histoire de l'art modernistes, commentaire rédigé au début des années 1970, au moment où Krauss rompait avec le formalisme de Greenberg ${ }^{42}$ :

La planéité que révère la critique moderniste peut bien avoir évacué la perspective spatiale mais elle lui en a substitué une temporelle, celle de l'histoire. C'est cette histoire que contemple, par exemple, la critique moderniste dans le vortex concentrique des bandes de Stella: une vue en perspective donnant derrière nous sur une succession décroissante de portes et de pièces traversées qui, parce qu'il n'est plus possible d'y pénétrer à nouveau, ne peuvent aujourd'hui se manifester que par l'intermédiaire d'une planéité diagrammatique.

Le Projet rend visibles les prémisses de ce credo moderniste, ses bases historicistes, contemporaines d'autres instances ayant contribué au même moment à l'autonomisation du champ de l'art: l'affirmation de la critique d'art, l'esthétique kantienne, la systématisation de l'histoire de l'art, la naissance de l'institution muséologique nationale. Ce tableau figure un moment charnière, celui de la première spatialisation en peinture du temps linéaire de l'histoire de l'art, appelé à devenir le principe explicatif de la peinture moderne et principalement de la peinture abstraite. Pour que la peinture finisse par s'enliser dans la conjoncture aporétique, dénoncée par Krauss en 1972 et nuancée plus récemment par Yve-Alain Bois, il faudra qu'une solution inverse à celle de Robert soit parvenue à s'imposer comme grand récit de la peinture moderniste, et que l'histoire de la peinture se soit élaborée en louangeant l'annulation de l'axe énonciatif de la perspective au profit de la seule affirmation, autonymique, autotélique, de la planéité opaque du support pictural.

C'est pourquoi, à l'heure d'une sortie hors du modernisme plastique, quand la peinture cherche encore à se situer par un oubli stratégiquement cultivé de l'histoire, le Projet demeure, autant que la Vue imaginaire, une ouvre importante pour saisir comment se sont noués les rapports douloureux de la peinture à l'histoire et comment ils rendent incontournables ceux de la peinture à un discours imaginaire de la fin, entendue ici dans le double sens d'une perpétuelle et fantasmatique imminence du terme et d'une finalité devenue problématique lors de la plus récente modernité de ce medium.

\section{N O TES}

1. Bois, Y.-A., «Painting: The Task of Mourning», Painting as Model, Cambridge, M.I.T., 1990, p.229.

2. Crimp, D., "The End of Painting", October no 16, 1981.

3. Cité dans Bois, op. cit., p. 243.

4. Barthes, R., «Réquichot et son corps ", L'Obvie et l'Obtus. Essais critiques III, Paris, Seuil, 1982, p. 211.

5. Cité dans Riout, D., La Peinture monochrome, Nîmes, Jacqueline Chambon, 1996, p. 51.

6. Taraboukine, N., " Du chevalet à la machine " [1923], Le Dernier Tableau, Paris, Champ libre, 1972, p. 41-42. 
7. De Duve, T., Nominalisme pictural, Paris, Minuit, 1984.

8. Bois, Y.-A., op. cit., p. 239.

9. Greenberg, C., «Modernist Painting", The New Art (éd. G.

Battcock), New York, Dutton, p. 66-77.

10. Riout, D., op. cit.

11. Pour une brève analyse de ce leitmotiv et une réflexion sur la boutade apocryphe de Delaroche, voir J. Lamoureux, «Delaroche et la mort de la peinture», Word and Image, mars-avril 1999.

12. Cité dans Seznec, J., Essais sur Diderot et l'Antiquité, Oxford, Oxford University Press, 1957, p. 44-45.

13. Poussin, N., Lettres et propos sur l'art (réunis par A. Blunt), Paris, Hermann, 1964, p. 118.

14. Garnier, N. et M.-C. Sahut, Le Louvre d'Hubert Robert, Paris,

Réunion des musées nationaux, 1979.

15. Diderot, D., Euvres esthétiques (éd. P. Vernière), Paris, Garnier, 1968, p. 760.

16. Peirce, C.S., Ecrits sur le signe (trad. G. Deledalle), Paris, Seuil, 1978, p. 151.

17. Caylus, A. C. Philippe, Recueils d'inscriptions égyptiennes, étrusques, grecques et romaines, Paris, 1752-1767, vol. IV (1761), p. 218-219.

18. Quatremère de Quincy, A., Lettres à Miranda sur le Déplacement des Monuments de l'Art de l'Italie (introd. et notes d'É. Pommier), Paris, Macula, 1989.

19. Quatremère de Quincy, A., Considérations morales sur la destination des ouvrages de l'art ou de l'influence de leur emploi, Paris, 1815, p. 55-57.

20. Corboz, A., Peinture militante et architecture révolutionnaire: à propos du thème du tunnel chez Hubert Robert, Bâle, Birkhaüser, 1978.

21. Diderot, D. et J.L.R. d'Alembert, « Peinture double », Encyclopédie ou Dictionnaire raisonné des sciences des arts et des métiers par une société de gens de lettres [1751-1780], Stuttgart, F. Frommann, 1966, tome XII, p. 279.

22. Alpers, S., The Art of Describing, Chicago, University of Chicago Press, 1983, p. 196-197.

23. Condorcet, J.-A.-N., Esquisse d'un tableau historique des progrès de l'esprit humain [1795], Paris, Garnier Flammarion, 1988. Sur

l'importance de l'ouvrage de Gibbon, The History of the Decline and Fall of the Roman Empire [1776-1788], et de la notion de déclin dans l'étude des arts au XVIII siècle, voir l'article de F. Haskell, "Gibbon and the History of art ", Past and Present in Art and Taste, New Haven, Yale University Press, 1987, p. 16-29.

24. Mortier, R., La Poétique des ruines en France, Genève, Droz, 1974. D'autres auteurs ont souligné des similitudes dans le discours des arts visuels, notamment A. Corboz. Corboz cite, dans le même esprit d'anticipation, la description par Cochin en 1757 de l'Église SainteGeneviève, commencée en 1755, telle qu'un archéologue de l'an 2355 la verrait. Saint-Aubin dessine une Vue prophétique de l'Église de SainteGeneviève en L'An 3000. Chambers réalise le projet pour le mausolée de Frédéric II (1752) sous forme de ruine. Ajoutons que l'Encyclopédie, dans sa présentation des monuments antiques, superpose un dessin en ruine et une vue en coupe. Enfin, une série de dix articles sur la peinture et l'architecture contemporaine, parue dans le Mercure de France de juillet 1755 à décembre 1766, emprunte le point de vue et la forme d'un mémoire préparé par des gens de lettres de l'an 2355. 25. Diderot, D., Ruines et Paysages. Salon de 1767, Paris, Hermann, 1995, p. 338. Diderot écrit à propos d'un tableau de Robert: «Les idées que les ruines évoquent en moi sont grandes. Tout s'anéantit, tout périt, tout passe. Il n'y a que le monde qui reste. Il n'y a que le temps qui dure. Qu'il est vieux ce monde. Je marche entre deux extrémités. De quelque part que je jette les yeux, les objets qui m'entourent m'annoncent une fin, et me résignent à celle qui m'attend". 26. L'exemple qui se présente tout de suite à l'esprit est L'An 2440 ou rêve s'il en fut (1770) de L.-S. Mercier (Bordeaux, Ducros, 1971) dans lequel l'auteur, s'étant endormi, se réveille, à la dite année dans une société égalitaire et pacifique où la morale et la philosophie sont à l'honneur. À lire Mercier, on n'a jamais le sentiment qu'un temps historique, événementiel, évolutif, a existé entre le temps de

l'énonciation et le moment anticipé et construit par l'auteur comme temps de l'énoncé.

27. Diderot, D. et J.L.R. d'Alembert, op. cit., tome IX, p. 707.

28. Mercier, L.-S., op. cit., p. 86.

29. Cité dans N. Garnier et M.-C. Sahut, op.cit., p. 34.

30. Condorcet, J.A.-N., op. cit., p. 259.

31. Cantarel-Besson, Y., La Naissance du Louvre, Paris, Réunion des musées nationaux, 1981, vol. II, p. 229. Je souligne.

32. Garnier, N. et M.-C. Sahut, op.cit., p. 32

33. Il existait trois fontes en bronze en France en 1796 mais le Traité de Tolentino devait bientôt lui céder l'original qui arriva en sol français dans une procession triomphale en juillet 1798 et fut exposé au Muséum de novembre 1800 à octobre 1815. Haskell, F. et N. Penny, Taste and the Antique, New Haven, Londres, Yale University Press, 1981, p. 148 et N. Garnier et M.-C. Sahut, op. cit., p. 60.

34. Haskell, F. et N. Penny, op. cit., p. 243.

35. Georgel, P., La Peinture dans la peinture, Dijon, Musée des beauxarts, 1982.

36. De Certeau, M., L'Écriture de l'histoire, Paris, Gallimard, 1975, p. $117-118$.

37. Foucault, M., Les Mots et les Choses, Paris, Gallimard, 1966, p. 137176. L'article «Galerie» de l'Encyclopédie, en renvoyant à celui sur les genres, insiste aussi implicitement sur les vertus classificatrices de la galerie.

38. Pomian, K., Collectionneurs, amateurs et curieux, Paris, Gallimard, 1987, p. 45

39. Ibid., p.59. Je souligne.

40. Marin, L., Détruire la peinture, Paris, Galilée, 1977, p. 62-66. À partir des travaux de Benveniste sur la nature des pronoms, Marin analyse la dénégation du dispositif énonciatif de la perspective dans La Rencontre des deux rois de Charles Le Brun, dénégation visant dans ce cas à accroître l'effet de vérité dans le déroulement latéral du récit. Voir Benveniste, É., Problèmes de linguistique générale, Paris, Gallimard, 1966, 2 vols.

41. On peut lire dans le rapport Varon que la galerie nationale conduira «insensiblement et par une marche graduelle l'histoire vivante de l'art, au plus période [sic] de sa grandeur et de sa force, c'està-dire à son terme. Sous ce point de vue, disparaissent bientôt ces distinctions ridicules d'histoire ou de genre, de paysages ou d'histoire: la nature n'ayant dit à personne qu'une danse de village fût une scène déplacée dans la galerie d'un peuple qui s'est imposé à lui-même le devoir d'honorer les vertus champêtres, et d'en préférer les douceurs". Cantarel-Besson, Y., op. cit., p. 228.

42. Krauss, R., "Un point de vue sur le modernisme», Regards sur l'art américain des années soixante (anthologie critique établie par C. Gintz), Paris, Territoires, 1979, p. 106. Krauss reproche au formalisme de faire dépendre "la signification d'une œuvre d'une comparaison avec des choses qui existent en dehors d'elles, [tout en considérant que] cette signification est tout entière présente dans la perception de l'œuvre seule". 